\title{
Sífilis Adquirida no Brasil: Análise retrospectiva de uma década (2010 a 2020)
}

\author{
Syphilis Acquired in Brazil: Retrospective analysis of a decade (2010 to 2020) \\ Sífilis adquirida en Brasil: Análisis retrospectivo de una década (2010 a 2020)
}

\author{
Iasmim Lima Menezes \\ ORCID: https://orcid.org/0000-0003-4101-1489 \\ Universidade Estadual da Paraíba, Brasil \\ E-mail: iasmimlimamenezes@gmail.com \\ Mayra Lucy de Macedo Targino \\ ORCID: https://orcid.org/0000-0002-9357-2678 \\ Universidade Estadual da Paraíba, Brasil \\ E-mail: mayralucy175@gmail.com \\ Ernani Canuto Figueirêdo Júnior \\ ORCID: https://orcid.org/0000-0002-1984-7477 \\ Universidade Estadual da Paraíba, Brasil \\ E-mail:ernanicfjunior@outlook.com \\ Flaviana Dornela Verli \\ ORCID: https://orcid.org/0000-0003-4241-4319 \\ Universidade Federal dos Vales do Jequitinhonha e Mucuri, Brasil \\ E-mail: flavianaverli@gmail.com \\ Sandra Aparecida Marinho \\ ORCID: https://orcid.org/0000-0002-5379-8779 \\ Universidade Estadual da Paraíba, Brasil \\ E-mail: san_mar2000@yahoo.com.br
}

\begin{abstract}
Resumo
Este estudo teve o propósito de realizar uma avaliação crítica dos dados disponíveis sobre sífilis adquirida no Sistema de Informação de Agravos de Notificação (SINAN), no período de 2010 a 2020. Verificou-se que até o ano de 2018, houve um aumento anual do registro de casos de sífilis, com queda no número de casos em 2019 e 2020 . Em 2020, houve redução equivalente a 1/3 do total de registros em relação ao ano anterior, provavelmente devido subnotificação decorrente da pandemia da Covid-19. O ano de 2018 foi o que apresentou maior número de casos notificados e a maior taxa de detecção (76,2 casos por 100 mil habitantes), sendo que a taxa de detecção nesse ano na região Sudeste $(82,5)$ superou a nacional. O sexo masculino foi mais acometido que o feminino, pelo comportamento de risco dos homens, contudo, com aumento crescente da infecção também nas mulheres, em decorrência da estabilidade do relacionamento, sem utilização do preservativo. Regiões mais desenvolvidas e populosas apresentaram mais notificações de casos. Apesar de a sífilis ser facilmente tratável, com administração de penicilina, ela ainda permanece um problema de saúde pública brasileira, sendo necessária mais atenção por parte das autoridades sanitárias, com maior investimento em campanhas de prevenção de infecções sexualmente transmissíveis. Essas, por sua vez, podem ser facilmente prevenidas com o uso de preservativos, que são distribuídos gratuitamente pelo Ministério da Saúde. Além disso, é importante difundir as complicações que a sífilis pode provocar quando não tratada, acarretando sua evolução, como recurso de alerta para a população.
\end{abstract}

Palavras-chave: Sífilis; Infecções por treponema; Doenças bacterianas sexualmente transmissíveis.

\begin{abstract}
This study had a propose of carrying out a critical evaluation of the available data on syphilis acquired in the Information System for Notifiable Diseases (SINAN), in the period from 2010 to 2020. It was verified that until the year 2018, there was an annual increase in the registration of syphilis cases, with a decrease in the number of cases in 2019 and 2020. In 2020 there was a reduction equivalent to $1 / 3$ of the total records, compared to the previous year, probably under-reporting due to Covid-19 pandemic. 2018 was the year with the highest number of reported cases and the highest detection rate (76.2 cases per 100 thousand inhabitants), with the detection rate for that year in the southeast region (82.5) surpassing the national one. The male gender was more affected than the female, due the risk behavior of men, however, with an increasing increase of infection also in women, due to the stability of the relationship, without use of the condom. More developed and populous regions had the highest number of case reports. Although syphilis is easily treatable, with penicillin administration, it still remains a Brazilian public health problem, requiring more attention from health authorities, with greater investment in campaigns to prevent sexually transmitted infections. These, in turn, can be easily prevented with the use of condoms, which are distributed free of charge by the Ministry of Health. In addition, it is important to spread the complications that syphilis can cause when not treated, causing its evolution, as a warning resource for the population.
\end{abstract}


Keywords: Syphilis; Treponemal infections; Sexually transmitted diseases bacterial.

\section{Resumen}

En este estudio tuvo el propósito de realizar una evaluación crítica de los datos disponibles sobre sífilis adquirida en el Sistema de Información de Enfermedades Notificables (SINAN), referido a los años 2010 a 2020. Se verificó que hasta el año 2018 hubo un aumento anual creciente en el registro de casos de sífilis, con una caída en el número de casos en 2019 y 2020. En 2020 hubo una reducción equivalente a 1/3 en el número de casos, en comparación con el año anterior, probablemente al subregistro debido a la pandemia Covid-19. 2018 fue el año con mayor número de casos reportados y mayor tasa de detección ( 76,2 casos por 100 mil habitantes), con la tasa de detección para ese año en la región sureste $(82,5)$ superando a la nacional. El género masculino se vio más afectado que el femenino, debido a la conducta de riesgo de los hombres, sin embargo, con un aumento creciente de la infección también en las mujeres, debido a la estabilidad de la relación, sin uso del condón. Las regiones más desarrolladas y pobladas tuvieron el mayor número de informes de casos. Aunque la sífilis es fácilmente tratable, con la administración de penicilina, sigue siendo un problema de salud pública brasileña, que requiere más atención por parte de las autoridades sanitarias, con una mayor inversión en campañas para prevenir las infecciones de transmisión sexual. Estos, a su vez, se pueden prevenir fácilmente con el uso de condones, los cuales son distribuidos gratuitamente por el Ministerio de Salud. Además, es importante difundir las complicaciones que la sífilis puede ocasionar cuando no se trata, lo que conlleva a su evolución, como recurso de alerta para la población.

Palabras clave: Sífilis; Infecciones por treponema; Enfermidades bacterianas de transmisión sexual.

\section{Introdução}

As infecções sexualmente transmissíveis (ISTs) ainda são grandes dificuldades e obstáculos para a saúde pública, e se não tratadas, apresentam consequências e efeitos graves, com implicações na saúde reprodutiva, além de o aumento de risco da transmissão do vírus da imunodeficiência humana (HIV), com manifestações clínicas mais agressivas e incomuns (Brasil, 2019).

Uma das ISTs mais comuns no mundo é a sífilis, com incidência de seis milhões de casos anuais (OMS, 2019). A transmissão da sífilis pode acontecer tanto por contato direto com as lesões, durante o ato sexual (sífilis adquirida), quanto por transmissão vertical (sífilis congênita), da mãe contaminada para o feto. Ocasionada pela bactéria Treponema pallidum, essa doença possui diferentes manifestações clínicas, sendo divididas em diferentes estágios ou fases, com sinais e sintomas específicos (CDC, 2017).

A sífilis adquirida pode ser classificada em sífilis primária, secundária, latente e terciária. Em relação ao tempo de evolução da doença, pode ser dividida em recente, até o primeiro ano de infecção, e tardia, após o primeiro ano de infecção (Brasil, 2016). A sífilis é uma doença infecto-contagiosa facilmente tratável, todavia, quando não tratada, pode se agravar, com consequências aos sistemas nervoso (neurossífilis), respiratório e gastrointestinal (Brasil, 2010). A neurossífilis e a sífilis ocular podem se desenvolver em qualquer etapa da sífilis, em meses ou anos após a contaminação (Etheridge et al., 2019; Lu et al., 2019).

O primeiro sinal da sífilis primária é caracterizado por uma úlcera, indolor e bem delimitada, conhecida como cancro duro, altamente infectante, podendo estar associada ao aparecimento de linfadenopatia regional (Brasil, 2010, 2020; Porterfield et al., 2020). As áreas mais comuns de manifestação são os órgãos genitais, podendo apresentar-se dolorosas em região anal (Richardson et al., 2020; Porterfield et al., 2020). As lesões podem aparecer também em outras regiões, como pele e boca, principalmente língua e lábio (Andrade et al., 2018; Brasil, 2020; Porterfield et al., 2020) e também palato mole (Andrade et al., 2018). Apresentam duração de três a oito semanas e desaparecimento espontâneo (Brasil, 2010, 2020; Etheridge et al., 2019).

A sífilis secundária surge de seis semanas a seis meses após o desaparecimento do cancro, em forma de lesões eritematosas (roséolas) em membros e nas regiões palmo-plantares. Nessa fase, ocorrem também sintomas inespecíficos como febre, cefaleia, mal-estar e fraqueza muscular, com desaparecimento em algumas semanas (Brasil, 2020). Além das lesões 
cutâneas, manifestações orais surgem em forma de máculas ou placas eritematosas (placa mucosa) ou ulcerações, principalmente em língua, gengiva e lábios, e menos comumente, em palato duro e comissura labial (Andrade et al., 2018).

Já a sífilis terciária surge após um intervalo de latência, podendo ocorrer entre um a 40 anos após o início da infecção, estando presente em $15 \%$ a $25 \%$ dos pacientes que não receberam tratamento. Nessa fase, ocorre destruição tecidual, com aparecimento de gomas sifilíticas, lesões indolores e destrutivas em mucosa e pele, ou outros tecidos, de evolução lenta (Brasil, 2020).

Para um diagnóstico definitivo e início do tratamento, exames complementares são imprescindíveis, como testes não treponêmicos e treponêmicos (Asselin et al., 2019). Os testes diagnósticos treponêmicos (FTA-Abs, TPHA, Elisa, entre outros) são específicos para a sífilis, podem ser convencionais (Brasil, 2015) ou rápidos.

O tratamento da sífilis consiste na administração de benzilpenicilina benzatina (penicilina G), padrão-ouro e medicamento de primeira escolha, iniciado imediatamente após o diagnóstico, em dose única (2,4 milhões UI, via intramuscular), em caso de sífilis recente; ou durante três semanas, na sífilis tardia ( 1 dose semanal de 2,4 milhões UI), com intervalos de uma semana entre as administrações, totalizando 7,2 milhões UI (Brasil, 2020). Em alérgicos à penicilina, são indicadas a ceftriaxona (1g, via endovenosa, 1x/dia), por 10 dias (Cao et al., 2017) e a doxiciclina (100mg, via oral, 12/12h), por 15 dias, para a sífilis recente, e por 30 dias, para a sífilis tardia (Brasil, 2020). Contudo, esses dois fármacos não devem ser administrados em gestantes (Cao et al., 2017; Brasil, 2020). Gestantes ou lactantes alérgicas à penicilina devem ser dessensibilizadas, e tratadas com penicilina. Se não for possível, o tratamento deve ser realizado com eritromicina (500mg, via oral, 6/6 horas), por 15 dias (sífilis recente), e por 30 dias (sífilis tardia) (Brasil, 2006). Após a terapia, o acompanhamento sorológico e clínico do paciente deve ser realizado por seis e 12 meses (Seña et al., 2017; Etheridge et al., 2019; Brasil, 2020).

Esse acompanhamento é essencial para relacionar o resultado da terapia e reconhecer uma possível reativação da infecção. A realização de testes não treponêmicos (VDRL-venereal disease research laboratory/ RPR- rapid plasmatic reagin) deve ser feita trimestralmente, durante um ano, em caso de pacientes HIV-positivos e aos seis e 12 meses, em HIV-negativos. O paciente HIV-positivo portador de sífilis apresenta manifestações clínicas mais agressivas, principalmente em relação aos problemas oculares e neurológicos. No decorrer do acompanhamento, os testes utilizados devem ser os mesmos que foram utilizados para o diagnóstico (Brasil, 2019, 2020). De acordo com Sena et al. (2017), o acompanhamento clínico e sorológico de HIV-positivos após o tratamento, deve ser trimestral durante um ano, e repetido após 24 meses.

Diante do exposto, considerando-se a importância e o impacto da sífilis para a saúde pública a proposta deste trabalho foi realizar uma avaliação crítica retrospectiva dos casos de sífilis adquirida notificados no Brasil, referente à última década (2009-2020).

\section{Metodologia}

A presente pesquisa consiste em um estudo ecológico, retrospectivo, descritivo com abordagem quantitativa (Pereira et al., 2018), sobre o número de casos de sífilis adquirida, notificados no Brasil entre os anos de 2010 a 2020.

A coleta de dados foi realizada no mês de novembro de 2020 e a mesma teve como fonte os dados disponíveis no Sistema de Informação de Agravos de Notificação (SINAN), base de dados disponibilizada pelo Departamento de Informática do Sistema Único de Saúde (DATASUS), sendo estes coletados através do site do DATASUS (http://www2.datasus.gov.br/DATASUS/index.php), conforme metodologia utilizada por Figueirêdo Júnior et al. (2020).

Todos os dados apresentados são de domínio e acesso público e foram obtidos através do acesso sequencial aos seguintes comandos disponíveis no site: >> "Informações em Saúde (TABNET)" >> "Epidemiológicas e Morbidade" >> "Doenças e Agravos de Notificação - 2007 em diante (SINAN)" >> "Sífilis congênita" >> "Dados regionais e nacionais". 
Salienta-se que, apesar de os dados aqui apresentados corresponderem aos casos de sífilis adquirida, os mesmos estão apresentados no sistema do DATASUS conjuntamente com os casos de sífilis congênita e não como uma categoria separada.

Para os casos de sífilis adquirida, foram coletados dados a nível nacional e por regiões geográficas, referentes às seguintes variáveis: casos notificados por ano de diagnóstico, taxa de detecção anual (por 100 mil habitantes) e casos separados por sexo e ano de diagnóstico. Todos os resultados estão apresentados através de estatística descritiva.

\section{Resultados e Discussão}

Entre os anos de 2010 e 2020, foi notificado um total de 783.544 casos de sífilis adquirida no Brasil. Considerando-se os dados por ano de diagnóstico, foi constatado um aumento crescente no número de casos entre os anos de 2010 e 2018, sendo esse último o que apresentou o maior número de casos (n=158.966) no período avaliado, com uma pequena redução do número de casos no ano de 2019. Interessantemente, no ano de 2020, houve uma diminuição drástica, que foi equivalente a mais de um terço de casos do ano anterior. Em 2020, os valores se assemelharam a 2014, sendo ligeiramente inferiores ao desse ano (Figura 1). Pode-se aventar que, devido a esse aumento sequencial e alarmante de casos até o ano de 2018, ações e medidas mais incisivas foram tomadas, com mais campanhas de prevenção e de tratamento, por parte das autoridades sanitárias, provocando maior conscientização da população para a prática do sexo seguro e também maior adesão ao tratamento, refletindo em 2019 e, felizmente, com maior ênfase, no ano de 2020. Deve-se também levar em consideração que, devido a pandemia do Coronavirus, houve uma redução das notificações no ano de 2020. Da mesma forma, no ano de 2018, os EUA apresentaram o maior número de casos notificados de sífilis desde 1991, com aumento de 13,3\% entre 2017 (101.584 casos) e 2018, com 115.045 casos (CDC, 2019).

Figura 1 - Casos de sífilis adquirida notificados no Brasil, por ano de diagnóstico no período de 2010-2020.

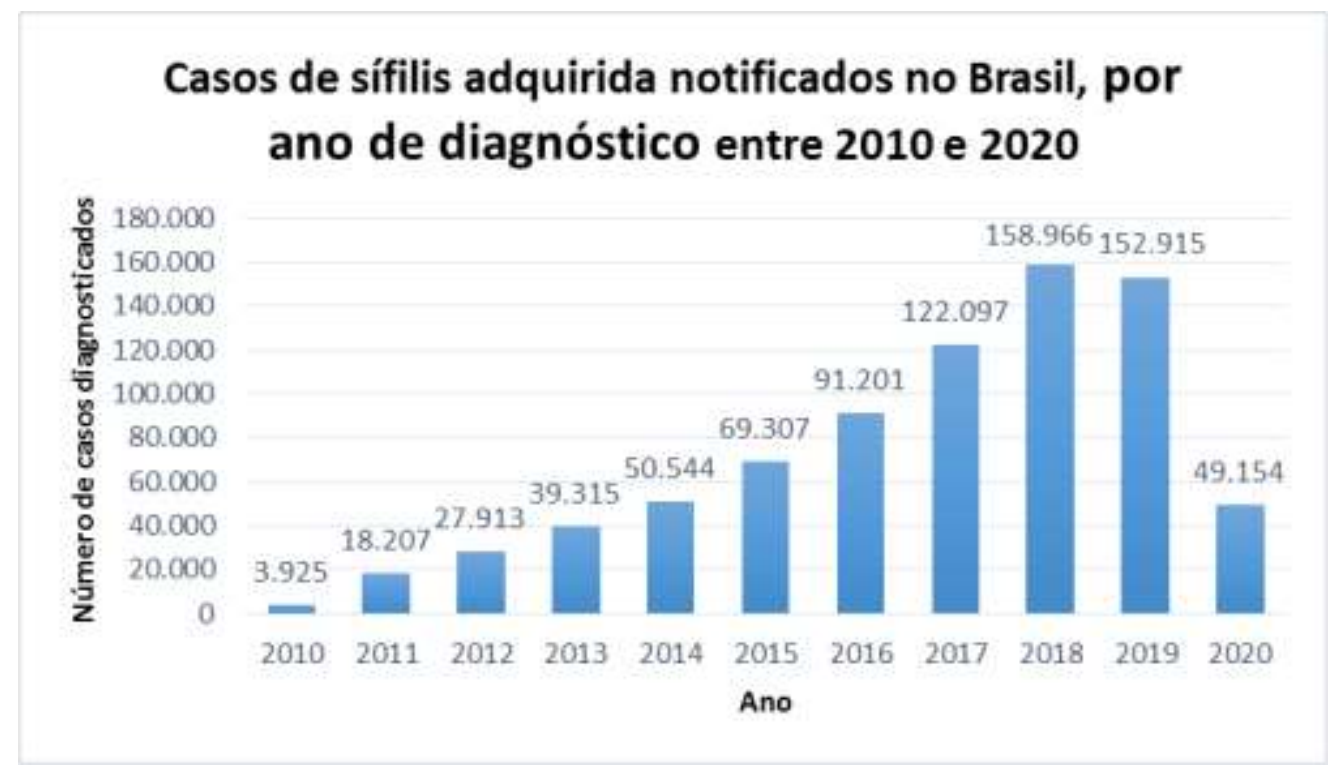

Fonte: Ministério da Saúde- Sistema de Informação de Agravos de Notificação (2020).

Considerando-se o quantitativo de casos notificados anualmente por regiões geográficas brasileiras (Figura 2), verificou-se que as regiões Sudeste e Sul foram, respectivamente, as que apresentaram os maiores números de casos em todos os anos avaliados. Exceção foi constatada apenas no ano de 2010, no qual a região Centro-Oeste ocupou o $2^{\circ}$ lugar. Em outro extremo, comparado às demais regiões, o Norte apresentou os menores números de casos em todos os anos avaliados. Importante ressaltar que a região sudeste é a região brasileira mais desenvolvida e populosa, e que também abriga grande 
concentração de profissionais da saúde, o que facilitaria a notificação. Já na região Norte, ocorre exatamente o oposto, por ser a menos populosa (Brasil, 2010) e consequentemente, concentrar um número bastante reduzido de profissionais, podendo haver provavelmente, subnotificação.

Figura 2 - Casos de sífilis adquirida notificados nas regiões geográficas, por ano de diagnóstico, no período de 2010-2020.

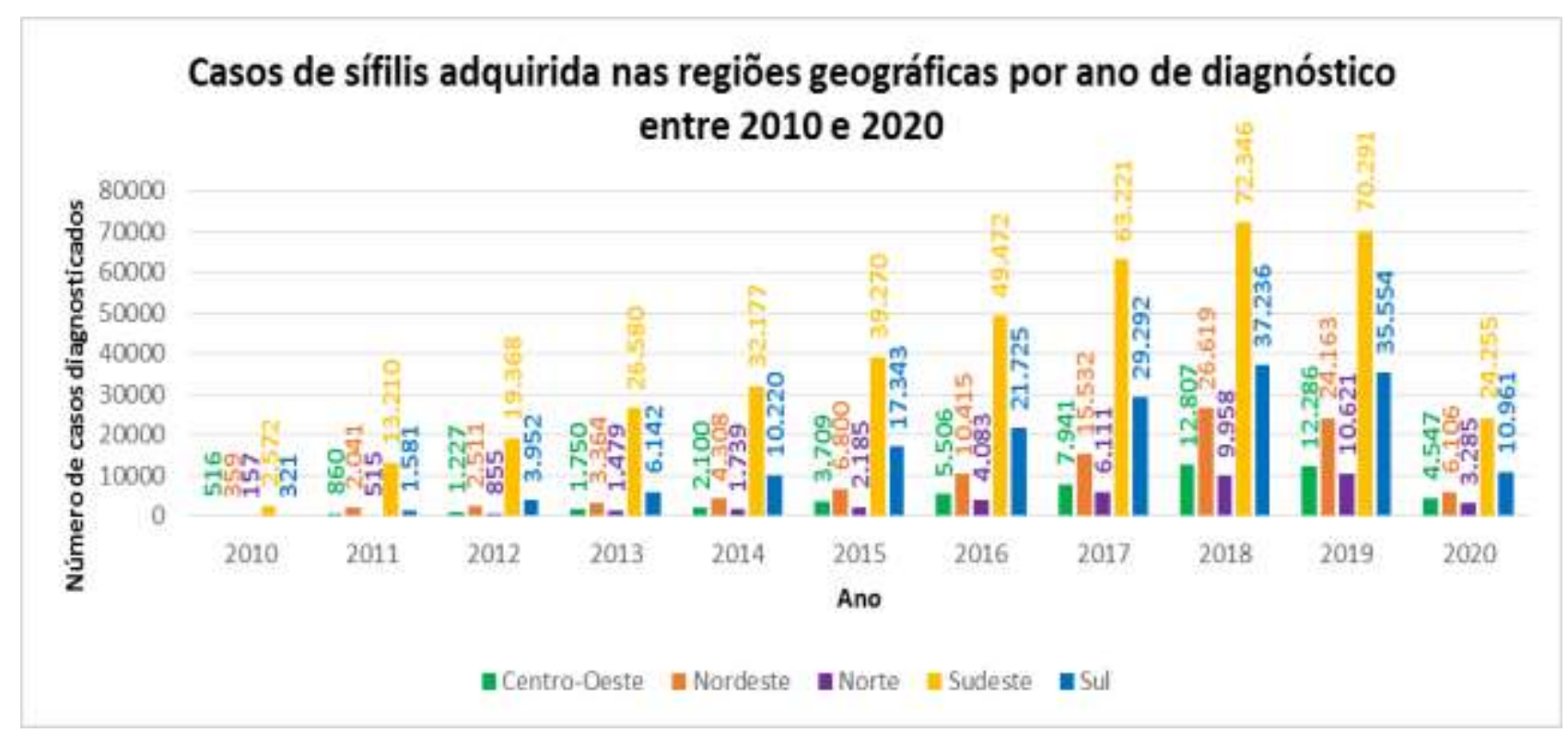

Fonte: Ministério da Saúde- Sistema de Informação de Agravos de Notificação (2020).

Considerando-se os dados referentes à taxa de detecção de casos de sífilis adquirida (por 100.000 habitantes), a nível nacional, foi constatado que a mesma manteve-se continuamente crescente entre os anos de 2010 e 2018, observando-se nesse último, a maior taxa de detecção de casos $(76,2)$ por 100 mil habitantes, seguido pelo ano de 2019, com taxa de 72,8 (Tabela 1). Nos EUA, também houve um aumento no índice de casos registrados de sífilis primária e secundária, com 9,4 casos por 100.000 habitantes em 2017, para 10,8 casos por 100.000 habitantes, em 2018 (CDC, 2019). Comparando-se, o Brasil apresentou sete vezes mais casos a cada 100 mil habitantes do que os EUA, nesse mesmo ano.

Considerando-se as regiões geográficas brasileiras, foi constatado que, no ano de 2010, o Centro-Oeste apresentou a maior taxa de detecção (por 100 mil habitantes) dentre as diferentes regiões, seguido pelo Sudeste, sendo ambos superiores à taxa nacional detectada para o mesmo ano. Por outro lado, no ano de 2011, essa relação se inverteu, passando o Sudeste a liderar a taxa de detecção e o Centro-Oeste a ocupar o segundo lugar. A partir daí, entre 2012 e 2014, o Sudeste prosseguiu com a liderança, seguido agora pela região Sul que, a partir de 2015 até 2019, passou a liderar, com maiores valores de detecção de casos por 100 mil habitantes, e o Sudeste passando para segunda posição, invertendo a situação. Destaca-se que nos anos de 2018 e 2019, apenas as taxas de detecção do Norte e Nordeste foram inferiores às médias nacionais, e em todo período avaliado, as taxas de deteç̧ão da região_Sudeste foram sempre superiores às taxas nacionais (Tabela 1). Salienta-se que, para o ano de 2020, os dados referentes a essa variável não se encontram disponíveis no sistema. 
Tabela 1 - Taxa de detecção de sífilis adquirida (por 100.000 habitantes) no período de 2010-2020 no Brasil e por regiões geográficas.

\begin{tabular}{lcccccc}
\hline Ano & Brasil & Centro-Oeste & Nordeste & Norte & Sudeste & Sul \\
\hline 2010 & 2,1 & 3,7 & 0,7 & 1,0 & 3,2 & 1,2 \\
2011 & 9,5 & 6,0 & 3,8 & 3,2 & 16,3 & 5,7 \\
2012 & 14,4 & 8,5 & 4,7 & 5,2 & 23,7 & 14,3 \\
2013 & 19,7 & 11,7 & 6,1 & 8,7 & 31,5 & 21,5 \\
2014 & 25,1 & 13,8 & 7,8 & 10,1 & 37,9 & 35,5 \\
2015 & 34,1 & 24,0 & 12,2 & 12,5 & 45,8 & 59,7 \\
2016 & 44,5 & 35,2 & 18,6 & 23,1 & 57,3 & 74,1 \\
2017 & 59,0 & 50,0 & 27,5 & 34,1 & 72,6 & 99,2 \\
2018 & 76,2 & 79,6 & 46,9 & 54,8 & 82,5 & 125,1 \\
2019 & 72,8 & 75,4 & 42,3 & 57,6 & 79,5 & 118,6 \\
2020 & ND & ND & ND & ND & ND & ND \\
\hline
\end{tabular}

ND: Dados não disponíveis. Fonte: Ministério da Saúde- Sistema de Informação de Agravos de Notificação (2020).

Considerando-se os casos detectados por sexo (Tabela 2) foi observada maior predominância de casos em indivíduos do sexo masculino, já que homens jovens apresentam maior predisposição a comportamentos de risco, em relação às mulheres (Castillo-Arcos et al., 2017), devido a certos comportamentos culturais como poligamia, parceiros casuais, uso de sexo comercial, e menor supervisão por parte dos pais, no caso de jovens (Adejumo, 2012; Morales-Mesa et al., 2014). Com o passar do tempo e consequente maior experiência sexual do jovem, ocorre uma redução progressiva quanto à intenção de uso de preservativos (Broaddus et al., 2011).

Destaca-se que, quanto ao sexo, considerando-se os dados disponíveis, a soma do número de casos (n=782.993) não condiz com o total de casos detectados no país $(n=783.544)$, com diferença de 551 casos, os quais não foram enquadrados/apresentados em outras categorias no sistema, podendo-se provavelmente os mesmos serem correspondentes a dados não registrados, perdidos ou ignorados, evidenciando falha na notificação.

Tabela 2 - Casos de sífilis adquirida por sexo e ano de diagnóstico no Brasil no período de 2010-2020.

\begin{tabular}{ccc}
\hline Ano & Sexo Feminino & Sexo Masculino \\
\hline & $\mathrm{n}(\%)$ & $\mathrm{n}(\%)$ \\
\hline 2010 & $1.468(0,47 \%)$ & $2.457(0,52 \%)$ \\
2011 & $7.219(2,30 \%)$ & $10.982(2,34 \%)$ \\
2012 & $10.872(3,46 \%)$ & $17.032(3,63 \%)$ \\
2014 & $15.868(5,05 \%)$ & $23.437(5,00 \%)$ \\
2015 & $20.060(6,38 \%)$ & $30.457(6,50 \%)$ \\
2016 & $27.534(8,76 \%)$ & $41.745(8,91 \%)$ \\
2017 & $37.048(11,79 \%)$ & $54.083(11,54 \%)$ \\
2018 & $50.285(16,00 \%)$ & $71.743(15,30 \%)$ \\
2019 & $64.144(20,41 \%)$ & $94.715(20,21 \%)$ \\
2020 & $61.399(19,54 \%)$ & $91.355(19,49 \%)$ \\
Total & $18.337(5,84 \%)$ & $30.753(6,56 \%)$ \\
\hline
\end{tabular}

Fonte: Ministério da Saúde- Sistema de Informação de Agravos de Notificação (2020). 
Considerando-se ainda os aspectos referentes ao sexo dos indivíduos acometidos por sífilis adquirida, de acordo com as regiões brasileiras, constatou-se que, de 2012 a 2020, as regiões Sudeste e Sul foram as que apresentaram em cada um desses anos consecutivos, respectivamente, o maior número de casos diagnosticados para ambos os sexos. Exceções a esse ranking foram verificadas nos anos de 2010 e 2011, onde as regiões que ocuparam o segundo lugar foram o Centro-Oeste em 2010, e o Nordeste em 2011 (Tabela 3). Para Empelen e Kok (2008), o fato de possuir um parceiro estável provocou atitudes menos favoráveis à utilização de preservativos, por parte dos jovens. Importante ressaltar que, no Brasil, a distribuição de preservativos ocorre de forma gratuita, fornecida pelo Ministério da Saúde (Brasil, 2020). Para isso, deve haver mais campanhas públicas preventivas e de promoção à saúde, principalmente voltada ao sexo masculino, para se evitar recontaminação e conscientização por parte dos homens (Cabral \& Valença, 2020). Contudo, ainda há certa dificuldade em se realizar ações preventivas voltadas aos homens, já que esses apresentam resistência à procura dos serviços da Atenção Primária em Saúde (Pereira et al., 2020), dificultando também o diagnóstico de uma IST já instalada e consequentemente o tratamento da mesma. Portanto, a valorização de campanhas educativas e eventos públicos podem contribuir como estratégia para a prevenção e diante da infecção, o tratamento oportuno da sífilis, tanto da mulher, quanto de seu parceiro (Muller et al., 2020).

Tabela 3 - Casos de sífilis adquirida por gênero e ano de diagnóstico nas regiões geográficas no período de 2010-2020.

\begin{tabular}{|c|c|c|c|c|c|}
\hline Ano & Centro-Oeste & Nordeste & Norte & Sudeste & Sul \\
\hline & & Sexo & Feminino & & \\
\hline 2010 & 233 & 167 & 70 & 857 & 141 \\
\hline 2011 & 350 & 1.008 & 219 & 4.981 & 661 \\
\hline 2012 & 480 & 1.203 & 386 & 7.170 & 1.633 \\
\hline 2013 & 739 & 1.888 & 692 & 9.979 & 2.570 \\
\hline 2014 & 780 & 2.330 & 771 & 11.994 & 4.185 \\
\hline 2015 & 1.293 & 3.664 & 928 & 14.085 & 7.564 \\
\hline 2016 & 1.880 & 5.260 & 1.731 & 18.670 & 9.507 \\
\hline 2017 & 2.944 & 7.533 & 2.541 & 24.098 & 13.169 \\
\hline 2018 & 4.548 & 10.871 & 4.182 & 27.688 & 16.855 \\
\hline 2019 & 4.393 & 9.511 & 4.089 & 27.403 & 16.003 \\
\hline \multirow[t]{2}{*}{2020} & 1.533 & 2.157 & 1.210 & 8.639 & 4.798 \\
\hline & & Sexo & Masculino & & \\
\hline 2010 & 283 & 192 & 87 & 1.715 & 180 \\
\hline 2011 & 508 & 1.033 & 296 & 8.225 & 920 \\
\hline 2012 & 747 & 1.307 & 469 & 12.191 & 2.318 \\
\hline 2013 & 1.011 & 1.472 & 787 & 16.595 & 3.572 \\
\hline 2014 & 1.317 & 1.972 & 968 & 20.173 & 6.027 \\
\hline 2015 & 2.404 & 3.134 & 1.257 & 25.172 & 9.778 \\
\hline 2016 & 3.597 & 5.148 & 2.350 & 30.770 & 12.218 \\
\hline 2017 & 4.976 & 7.982 & 3.570 & 39.092 & 16.123 \\
\hline 2018 & 8.233 & 15.733 & 5.774 & 44.596 & 20.379 \\
\hline 2019 & 7.848 & 14.611 & 6.527 & 42.839 & 19.530 \\
\hline 2020 & 2.973 & 3.943 & 2.074 & 15.603 & 6.160 \\
\hline
\end{tabular}

Fonte: Ministério da Saúde- Sistema de Informação de Agravos de Notificação (2020).

\section{Conclusão}

Apesar de possuir uma simples forma de prevenção e de ser facilmente tratada, a sífilis é uma doença que ainda permanece emergente no país. Dentre os anos de 2010 e 2020, o ano de 2018 foi o que apresentou o maior número de casos, com maior concentração na região Sudeste, a mais populosa. Em contrapartida, no ano de 2020, o número de casos foi 3,1 vezes_menor que em 2019, o que seria justificado pela subnotificação ocorrida no período pandêmico da COVID-19, sendo necessários novos estudos. É válido ressaltar que ambos os sexos apresentaram riscos crescentes de contaminação, entretanto, o sexo masculino foi o mais acometido, tendo em vista o comportamento cultural dos homens jovens, que possuem inclinação a atitudes de risco. Em relação às mulheres, o aumento no risco de contaminação pode estar associado à confiança no parceiro 
e estabilidade da relação, que tem como consequência a não utilização de preservativos. As autoridades sanitárias devem se atentar mais para as infecções sexualmente transmissíveis, como a sífilis, procurando incentivar a utilização de preservativos masculinos, como forma de prevenção e proteção, tanto para homens como para mulheres. Além disso, é importante difundir, como forma de alerta para a população, as consequências decorrentes do não tratamento de um portador de sífilis adquirida.

\section{Referências}

Araújo, M. S. T. \& Abib, M. L. V. S. (2003). Atividades experimentais no ensino de física: diferentes enfoques, diferentes finalidades. Rev. Bras. Ens. Fís., 25(2), 176-194.

Adejumo, A. O. (2012). Influence of psycho-demographic factors and effectiveness of psycho-behavioral interventions on sexual risk behavior of in-school adolescents in Ibadan, Nigeria. Ife Psychol., 20(1), 1-18.

Andrade, R. S. et al. (2018). Oral findings in secondary syphilis. Med. Oral Patol. Oral Cir. Bucal., 23(2), e138-e143. 10.4317/medoral.22196.

Asselin, C., Ekindi, N., Carignan, A., \& Richard, P. O. (2019). Gummatous penile syphilis. IDCases, 18, e00589. 10.1016/j.idcr.2019.

Brasil. Instituto Brasileiro de Geografia e Estatística. Censo Demográfico 2010. 2010.

Brasil. Ministério da Saúde. Sífilis: o que é, causas, sintomas, tratamento, diagnóstico e prevenção. http://saude.gov.br/saude-de-a-z/sifilis.

Brasil. Ministério da Saúde. Secretaria de Atenção Primária à Saúde (SAPS). Principais ações em saúde para prevenção da gravidez na adolescência. https://aps.saude.gov.br/noticia/7196.

Brasil. Ministério da Saúde. Secretaria de Vigilância em Saúde. (2019). Boletim epidemiológico sífilis 2019. http://www.aids.gov.br/pt-br/pub/2019/boletimepidemiologico-sifilis-2019.

Brasil. Ministério da Saúde. Secretaria de Vigilância em Saúde. Diretrizes para o controle da sífilis congênita: Manual de bolso. (2a ed.).

Brasil. Ministério da Saúde. Secretaria de Vigilância em Saúde. (2016) Manual técnico para diagnóstico da sífilis. http://www.aids.gov.br/ptbr/pub/2016/manual-tecnico-para-diagnostico-da-sifilis.

Brasil. Ministério da Saúde Secretaria de Vigilância em Saúde. (2015). Protocolo clínico e diretrizes terapêuticas para atenção integral às pessoas com infecções sexualmente transmissíveis (IST). http://www.aids.gov.br/pt-br/pub/2015/protocolo-clinico-e-diretrizes-terapeuticas-para-atencao-integral-pessoascom-infeccoes.

Brasil. Secretaria de Vigilância em Saúde/ Ministério da Saúde. Sífilis estratégias para diagnóstico no Brasil. http://bvsms.saude.gov.br/bvs/publicacoes/sifilis_estrategia_diagnostico_brasil.pdf.

Broaddus, M. R., Schmiege, S. J., \& Bryan, A. D. (2011). An expanded model of the temporal stability of condom use intentions: gender-specific predictors among high-risk adolescents. Ann. Behav. Med., 42, 99-110.

Cao Y. et al. (2017). A multicenter study evaluating ceftriaxone and benzathine penicillin G as treatment agents for early syphilis in Jiangsu, China. Clin. Infect. Dis., 65(10), 1683-1688.

Cabral, C. L.\& Valença, J.G. (2020). Perfil epidemiológico da sífilis e o papel do Dentista. Res. Soc. Develop, 9(8), e52985387.

Castillo-Arcos, L. C., et al. (2017). Age, Gender and Resilience in Sexual Risk Behavior of STI among adolescents in Southern Mexico. Enf. Global, 45, 178186. http://dx.doi.org/10.6018/eglobal.16.1.234921

Centers for Disease Control and Prevention (CDC). Sexually Transmitted Diseases (STDs) - Syphilis - CDC Fact Sheet. https://www.cdc.gov/std/syphilis/stdfact-syphilis.htm.

Centers for Disease Control and Prevention (CDC). Sexually Transmitted Disease Surveillance 2018: Syphilis. https://www.cdc.gov/std/stats18/syphilis.htm.

Empelen, P., \& Kok, G. (2008). Actio-specific cognitions of planned and preparatory behaviors of condom use among dutch adolescents. Arch. Sex. Behav., $37,626-640$.

Etheridge, T. et al. (2019). Ocular syphilis: clinical manifestations and treatment course. WMJ. 118(4), 191-195.

Figueirêdo Júnior, E. C. et al. (2020). Perfil epidemiológico dos casos de Aids notificados no Brasil entre os anos de 2009 a 2019. Res. Soc. Develop., 9(9), e302997233.

Lu, Y. et al. (2019). Clinical prediction and diagnosis of neurosyphilis in HIV-negative patients: a case-control study. BMC Infect. Dis., 19(1), 1017. $10.1186 / \mathrm{s} 12879-019-4582-2$.

Morales-Mesa, S., Arboleda-Álvarez, O., \& Segura-Cardona, A. (2014). Las prácticas sexuales de riesgo en poblaciones universitarias. Rev. Salud Publica, $16(1), 27-39$.

Muller, E. A. et al. (2020). Estratégias utilizadas por Enfermeiros frente ao teste rápido reagente para sífilis em gestantes. Res. Soc. Develop., 9(8), e685986055. 
Research, Society and Development, v. 10, n. 6, e17610611180, 2021

(CC BY 4.0) | ISSN 2525-3409 | DOI: http://dx.doi.org/10.33448/rsd-v10i6.11180

Ohta, A. et al. (2018). Secondary syphilis with pulmonary involvement. Inter. Med., 57(1), 121-126.

Pereira, A. S. et al. (2018). Metodologia da pesquisa científica. UFSM. https://repositorio.ufsm.br/bitstream/handle/1/15824/Lic_Computacao_MetodologiaPesquisa-Cientifica.pdf?sequence $=1$.

Pereira, E. L. et al. (2020). Planejamento estratégico situacional como ferramenta para promoção da saúde do homem: relato de experiência. Res Soc Develop, $9(9)$, e668997821.

Porterfield, C. et al. (2020). Primary syphilis presenting as a chronic lip ulcer. Cureus, 12(2), e7086. 10.7759/cureus.7086.

Richardson, D. et al. (2020). Primary syphilis in men causes painful anogenital lesions and serology is not always helpful. Sex. Transm. Infect. sextrans-2020054495.10.1136/sextrans-2020-054495.

Seña, A. C. et al. (2017). Rate of decline in nontreponemal antibody titers and seroreversion after treatment of early syphilis. Sex. Transm. Dis., 44(1), 6-10. 